\title{
Phytoprotection
}

\section{Le potentiel de l'écologie chimique dans la lutte contre les insectes nuisibles}

\section{J.N. McNeil et J. Delisle}

Volume 74, numéro 1, 1993

URI : https://id.erudit.org/iderudit/706034ar

DOI : https://doi.org/10.7202/706034ar

Aller au sommaire du numéro

Éditeur(s)

Société de protection des plantes du Québec (SPPQ)l

ISSN

0031-9511 (imprimé)

1710-1603 (numérique)

Découvrir la revue

Citer cet article

McNeil, J. \& Delisle, J. (1993). Le potentiel de l'écologie chimique dans la lutte contre les insectes nuisibles. Phytoprotection, 74(1), 29-39.

https://doi.org/10.7202/706034ar
Résumé de l'article

En nature, plusieurs organismes vivants émettent des substances chimiques médiatrices (infochimiques) lorsqu'ils créent des liens complexes et variés entre les individus de la même espèce ou d'espèces différentes. Dans cet article, nous décrivons comment certains insectes utilisent les substances infochimiques pour la recherche d'un partenaire sexuel, de sources de nourriture ou d'un lieu favorable pour la ponte. De plus, nous discutons de l'intérêt de bien comprendre les fondements de l'écologie chimique afin de promouvoir le développement et l'utilisation de méthodes de lutte plus efficaces contre les insectes nuisibles et plus acceptables pour l'environnement. 


\title{
Le potentiel de l'écologie chimique dans la lutte contre les insectes nuisibles
}

\author{
Jeremy N. McNeil ${ }^{1}$ et Johanne Delisle ${ }^{2}$
}

Reçu 1992-11-18; accepté 1993-05-11

En nature, plusieurs organismes vivants émettent des substances chimiques médiatrices (infochimiques) lorsqu'ils créent des liens complexes et variés entre les individus de la même espèce ou d'espèces différentes. Dans cet article, nous décrivons comment certains insectes utilisent les substances infochimiques pour la recherche d'un partenaire sexuel, de sources de nourriture ou d'un lieu favorable pour la ponte. De plus, nous discutons de l'intérêt de bien comprendre les fondements de l'écologie chimique afin de promouvoir le développement et l'utilisation de méthodes de lutte plus efficaces contre les insectes nuisibles et plus acceptables pour I'environnement.

McNeil, J. N., and J. Delisle. 1993. The potential of chemical ecology in insect pest management. PHYTOPROTECTION 74: 29-39.

Infochemicals are important in modulating a large number of both intra- and interspecific interactions in nature and in this paper we examine examples of their use by insects in the location of mates, food and suitable oviposition sites. Furthermore, we consider how an understanding of chemical ecology can be used to develop more efficient and ecologically acceptable pest management programs.

\section{INTRODUCTION}

Du point de vue écologique, il y a plusieurs désavantages à utiliser les insecticides chimiques pour lutter contre les insectes nuisibles des milieux agricoles et forestiers. Non seulement ces produits sont toxiques et de faible sélectivité, c'estdire-à que plusieurs espèces non visées, incluant l'homme, ne sont pas à l'abri de leurs effets nocifs, mais ils sont aussi inefficaces contre plusieurs insectes nuisibles devenus résistants (Metcalf 1980). Face à une telle situation, il est important que la lutte contre les insectes nuisibles soit réalisée à partir de produits plus sécuritaires pour la santé humaine et plus

1. Département de biologie, Université Laval, Québec (Québec) Canada G1K 7P4

2. Forêts Canada, Région du Québec, 1055, rue du P.E.P.S, Sainte-Foy (Québec)

Canada G1V 4C7 respectueux de l'environnement. C'est dans ce contexte que le concept de la lutte intégrée a été proposé comme étant la solution la plus valable. Bien que cette idée ne soit pas nouvelle (Rabb et Guthrie 1970), nous sommes encore aujourd'hui fort dépendants de l'usage des insecticides chimiques pour minimiser les pertes pré- ou post-récolte causées par les insectes ravageurs. Si le passage de la lutte strictement chimique à une approche plus équilibrée et intégrée pour la lutte contre les insectes nuisibles, les mauvaises herbes ou les agents pathogènes se fait lentement, ceci est attribuable en partie à une connaissance insuffisante des systèmes biologiques par rapport auxquels une intervention plus efficace est désirée.

Au cours des dernières années, l'écologie chimique, définie par Feeny (1992) comme étant: "l'étude des relations chimiques entre un organisme et son milieu, incluant tout autre organisme vi- 
vant", a connu un essor considérable si I'on en juge par la publication récente de plusieurs ouvrages traitant aussi bien de ces aspects fondamentaux qu'appliqués (Barbosa et Letourneau 1988; Heinrichs 1988; Hunter et al. 1992; Jutsum et Gordon 1989; Metcalf et Metcalf 1992; Muirhead-Thomson 1991; Price et al. 1992; Ridway et al. 1990; Roitberg et Isman 1992; Smith 1989). C'est dans cette perspective que nous examinerons les rôles joués par les composés infochimiques chez les insectes dans la quête d'un partenaire sexuel, la recherche d'un site convenable pour la prise alimentaire et la ponte, ainsi que dans les multiples relations entre phytophages et ennemis naturels. Aussi, nous emprunterons à Dicke et Sabelis (1988), I'utilisation du terme "infochimique" pour définir les substances chimiques médiatrices émises par un organisme vivant lors d'une relation intra- ou interspécifique et qui provoquent chez celui qui les détecte, un changement comportemental ou physiologique susceptible de favoriser l'un ou l'autre des organismes, ou les deux. Le même infochimique peut ainsi avoir divers rôles, selon la relation qui s'établit entre l'émetteur et le récepteur. Par exemple, un infochimique peut attirer à la fois un partenaire sexuel, agissant ainsi comme phéromone sexuelle (relation intraspécifique) et des entomophages, et se nommer dans ce dernier cas une kairomone (relation interspécifique). Enfin, nous discuterons, à l'intérieur de chacune des relations infochimiques décrites, des possibilités nouvelles qu'offre l'écologie chimique dans la lutte contre les insectes ravageurs.

Ce n'était pas notre intention de présenter une revue exhaustive du sujet; d'ailleurs, certains thèmes importants, comme celui du rôle des infochimiques dans la prospection alimentaire des larves en vue de la prise de nourriture (e.g. Chapman et Bernays 1989), n'ont pas été discutés. Nous avons préféré nous en tenir à quelques exemples qui démontrent bien l'importance de l'écologie chimique à différents niveaux de la chaîne trophique. Nous tenons aussi à souligner que c'est par intérêt personnel que nous avons accordé une attention particulière aux Lépidoptères et non parce que les relations impliquant des infochimiques n'existent pas chez d'autres ordres d'insectes.

\section{L'ATTRACTION ET LE CHOIX D'UN PARTENAIRE SEXUEL}

Les phéromones intervenant dans les comportements précopulatoires ont été observées chez divers ordres d'insectes. Chez la majorité des espèces étudiées, ce sont surtout les femelles, sexuellement matures, qui émettent l'infochimique servant à orienter de plus ou moins loin le vol du partenaire sexuel. Cependant, les mâles de certaines espèces peuvent aussi émettre des phéromones sexuelles agissant à longue distance (Hardee et al. 1969; Kuba and Sokei 1988). De plus, chez d'autres espèces, les phéromones sexuelles peuvent être produites aussi bien par le mâle que la femelle, mais dans ces cas, chacun l'émet à des temps différents pendant la nuit (Landolt et Heath 1990; Willis et Birch 1982). Sauf pour les espèces mentionnées ci-haut, il existe aussi des phéromones sexuelles mâles qui agissent à courte distance et qui sont relâchées pendant la période de cour, grâce à des glandes ou des organes spéciaux, situés à différents endroits du corps. Dépendant des espèces, la phéromone sexuelle mâle peut jouer différents rôles (Birch et al. 1990; Fitzpatrick et McNeil 1988). Chez certains insectes, elle inhibe les déplacements de la femelle et facilite ainsi l'accouplement, alors que chez d'autres, elle inhibe plutôt l'appétence des mâles congénères, réduisant ainsi la compétition intrasexuelle. Chez les espèces apparentées phylogénétiquement, la phéromone sexuelle peut agir en tant que mécanisme d'isolement reproducteur. II y a aussi des exemples concrets démontrant qu'elle peut aider les femelles à mieux juger de la qualité des mâles, en tant que géniteurs potentiels, car ceux qui l'émettent en faibles quantités ou pas du tout ont vraisemblablement un succès d'accouplement inférieur à ceux qui en sont mieux pourvus (Fitzpatrick et McNeil 1988; Phelan et Baker 1986; Pivnick et al. 1992; Royer et McNeil 1992). De plus, le fait de choisir un mâle de qualité supérieure augmenterait le succès reproducteur de la femelle en favorisant la production d'une descen- 
dance plus forte (Phelan et Baker 1986) ou plus nombreuse (Royer et McNeil 1993), ou encore mieux protégée contre les prédateurs, grâce aux substances de défense transférées par le mâle au moment de l'accouplement (Dussourd et al. 1988).

Bien que les phéromones sexuelles agissant à courte distance (généralement celles émises par les mâles) présentent peu d'intérêt en recherche appliquée, il en est autrement des phéromones sexuelles à longue distance qui sont produites normalement par les femelles. La facilité avec laquelle ces phéromones peuvent être synthétisées a contribué à leur emploi efficace dans divers domaines de la recherche entomologique. Certains chercheurs les utilisent comme outils de surveillance ou de détection, permettant ainsi de suivre l'évolution temporelle et spatiale des populations d'insectes nuisibles (Wall 1990), alors que d'autres les utilisent pour des fins de contrôle, soit direct, par piégeage massif des papillons mâles (Lanier 1990) ou indirect, par inhibition de l'accouplement au moyen de la confusion sexuelle (Cardé 1990). Également, lorsque la phéromone sexuelle est combinée à un insecticide chimique, elle peut être utilisée comme attracticide spécifique, réduisant ainsi l'impact négatif des insecticides chimiques sur les ennemis naturels des espèces cibles ou de toute autre espèce présente dans le milieu (Haynes et al. 1986a). La même technique peut servir aussi à tester rapidement le degré de résistance des populations d'insectes aux insecticides chimiques (Haynes et al. 1986b, 1987).

Cependant, pour que le rendement des phéromones de synthèse soit optimal, en particulier concernant leur capacité à compétitionner avec les phéromones de femelles vierges, il est nécessaire de bien connaître leur composition chimique ainsi que la proportion exacte des différents composés constituant le bouquet phéromonal. Par exemple, chez la tordeuse à bandes obliques [Choristoneura rosaceana (Harris)] [Lepidoptera: Tortricidae], une étude récente a démontré que l'efficacité de la phéromone de synthèse pouvait être considérablement améliorée par l'addition de composés mineurs (Delisle 1992). Cependant, même en présence du meilleur mélange, les femelles vierges se sont montrées toujours plus attirantes, suggérant que la composition du leurre synthétique $n^{\prime}$ est pas encore parfaitement au point (Delisle 1992). En plus de préparer des formulations et des concentrations exactes de la phéromone de synthèse, il est important aussi de bien comprendre l'influence des divers agents abiotiques (humidité, température, photopériode, précipitations, vitesse du vent) et biotiques (âge, capacité d'accouplement, infections) sur les rythmes d'émission et de réception de la phéromone sexuelle (McNeil 1991). De telles informations sont non seulement très utiles pour choisir la période et l'endroit convenant le mieux au déploiement des pièges à phéromone, mais aussi pour mieux évaluer le degré de compétition entre les femelles vierges et les leurres synthétiques utilisés dans les programmes de lutte. Nous devons aussi mieux comprendre les mécanismes intervenant dans le choix du partenaire sexuel par la femelle ainsi que dans la compétition intrasexuelle des mâles afin d'interpréter correctement les captures obtenues dans les pièges à phéromone, soit pour des fins de dynamique de populations ou pour augmenter les possibilités de lutte par confusion sexuelle (McNeil 1992). Par exemple, nous aurions une fausse indication de l'importance de la population en voie de reproduction si les mâles capturés dans les pièges à phéromone n'étaient que ceux rejetés par les femelles comme partenaire sexuel.

\section{LA RECHERCHE DES SOURCES DE NOURRITURE PAR LES PAPILLONS}

Les adultes de certaines espèces de Lépidoptères ne se nourrissent jamais ou presque pas. Chez d'autres espèces, la prise alimentaire est essentielle aux deux sexes et, en l'absence de celle-ci, les individus peuvent vivre moins longtemps ou être incapables de voler et de s'accoupler, affectant ainsi leur succès de reproduction. Chez les Lépidoptères qui s'alimentent, le nectar est la principale source de nourriture et, même si certains sont capables d'extraire les nutriments du pollen (Gilbert 1972), ils sont dans un cas 
comme dans l'autre fort dépendants de la présence des fleurs. Tout comme les mâles qui utilisent les phéromones pour repérer les femelles réceptives sexuellement, les femelles des papillons de nuit utilisent les composés odoriférants des fleurs pour trouver leurs plantes-hôtes (Haynes et al. 1991; Wiesenborn et Baker 1990). Ceci est particulièrement avantageux lorsque les fleurs ne sont pas distribuées uniformément dans I'habitat ou qu'elles fleurissent à des temps différents pendant la saison.

L'utilisation des composés odoriférants des fleurs comme méthode potentielle de lutte contre les insectes nuisibles a été démontrée récemment chez la faussearpenteuse du chou [ Trichoplusia ni (Hübner)] [Lepidoptera: Noctuidae] (Landolt et al. 1991). A l'aide d'une solution de sucre contenant un insecticide et du phénylacétaldéhyde, le plus attirant des volatiles produits par les fleurs d'un arbuste, lesquelles sont visitées par cet insecte (Haynes et al. 1991), la mortalité des adultes s'est élevée à $44 \%$ chez les mâles et à $66 \%$ chez les femelles comparée à 5 et $6 \%$ chez les témoins respectifs.

\section{LE CHOIX D'UN SITE CONVENABLE POUR LA PONTE}

II est évident qu'en nature, les insectes phytophages ne pondent pas leurs oeufs de façon aléatoire sur toutes les plantes qu'ils rencontrent dans I'habitat. Certaines femelles Lépidoptères sont effectivement capables de détecter à distance les arômes émanant des plantes-hôtes et utilisent ces stimuli pour choisir celles qui semblent les plus convenables pour la ponte (Haynes et Baker 1989; Landolt 1989; Ramaswamy 1988; Tingle et Mitchell 1991; Tingle et al. 1990). De façon similaire, les odeurs perçues à courte distance ou par contact direct sont d'autres stimuli intervenant dans la reconnaissance des plantes-hôtes par les femelles (Delisle et al. 1989; Renwick 1989; Renwick et al. 1992; van Loon et al. 1992). Chez I'Heliothis virescens (F.) [Lepidoptera: Noctuidae], il a été démontré, dans un tunnel aérodynamique, que les femelles répondaient moins bien aux odeurs de tabac (Nicotiana tabacum L.) ou de coton
(Gossypium hirsutum L.) quand ces dernières étaient mélangées à celles des plantes non-hôtes (Tingle et Mitchell 1991). Même si dans cette expérience, certaines femelles ont pu être attirées par le mélange d'odeurs des plantes-hôtes et non-hôtes, très peu d'entre elles s'y sont posées et y ont pondu. Des comportements similaires ont aussi été observés lorsque la réponse des femelles aux odeurs émanant des variétés susceptibles et résistantes de tabac a été testée (Tingle et al. 1990). Ces résultats sont encourageants car ils laissent entrevoir la possiblité de produire par manipulations génétiques, des variétés de plantes qui seront exemptes d'attractifs odorants ou qui en produiront mais à des niveaux ne favorisant pas l'attraction. Cependant, cette voie ne sera pas utile avant que la nature des infochimiques impliqués dans le comportement de ponte soit corinue. Une autre méthode potentiellement intéressante pour la lutte est l'incorporation d'odeurs de plantes non-hôtes à des plantes de culture, soit par manipulations génétiques ou comme l'a déjà proposé Visser (1986), par diversification des cultures.

Le choix d'un site convenable pour la ponte peut aussi être influencé par les odeurs produites par les femelles Lépidoptères elles-mêmes. Les femelles du Pieris rapae (L.) [Lepidoptera: Pieridae] et $P$. brassicae (L.) [Lepidoptera: Pieridae] incorporent dans leurs oeufs une phéromone composée de substances volatiles et non volatiles, produites vraisernblablement par les glandes accessoires, et qui a pour effet de prévenir la ponte des congénères (Schoonhoven 1990; Schoonhoven et al. 1990). D'autres observations ont démontré que la pulvérisation d'un extrait de ces oeufs sur des plantes de crucifères rendaient ces dernières moins attirantes aux femelles comparées à des plantes témoins (Schoonhoven 1990; Schoonhoven et al. 1990). De plus, quelle que soit l'espèce de piéride, la phéromone a inhibé aussi bien la ponte des femelles de l'autre espèce que celle des congénères (Schoonhoven 1990; Schoonhoven et al. 1990), démontrant ainsi sa capacité d'agir comme kairomone.

Bien qu'il existe, en lutte intégrée, un certain potentiel pour les phéromones 
d'anti-oviposition, tel que démontré chez la mouche du cerisier (Rhagoletis cerasi L.) [Diptera: Tephritidae] (Aluja et Boller 1992), certaines difficultés doivent cependant être résolues avant de pouvoir les utiliser sur une plus grande échelle. D'une part, la nature chimique de ces phéromones n'est pas connue à l'exception d'une seule (Hurter et al. 1987) et d'autre part, comme elles sont solubles dans l'eau, leur persistance et leur efficacité peuvent être affectées par certaines conditions climatiques. Par exemple, chez la mineuse virgule de la luzerne (Agromyza frontella Rondani ) [Diptera: Agromyzidae] la phéromone $d^{\prime}$ 'anti-oviposition ne persiste pas plus de $48 \mathrm{~h}$ en nature (Quiring et McNeil 1984), alors que chez la mouche de la pomme [Rhagoletis pomonella (Walsh)] [Diptera: Tephritidae] la pluie affecte son efficacité (Averill et Prokopy 1987). De plus, même si la dispersion des femelles augmente lorsqu'elles rencontrent des plantes marquées par la phéromone d'anti-oviposition (Klijnstra et Schoonhoven 1987; Quiring et McNeil 1987; Roitberg et al. 1982, 1984), elles finissent par les accepter comme sites de ponte à mesure que la disponibilité des plantes non-marquées se fait de plus en plus rare. II semble donc que l'utilisation des phéromones d'anti-oviposition dépendra de l'espèce cible et des conditions climatiques.

\section{LE RÔLE DES INFOCHIMIQUES DANS LA RECHERCHE DES HÔTES PAR LES ENTOMOPHAGES}

Tout comme les phytophages qui utilisent les arômes des plantes-hôtes pour trouver des sites convenables pour la ponte, la recherche d'un hôte par un insecte parasitoïde, ou d'une proie par un prédateur, est étroitement reliée à l'émission d'infochimiques par l'hôte ou la proie. Par exemple, un entomophage peut être attiré par la phéromone sexuelle émise par la femelle Lépidoptère (Noldus et al. 1991a, 1991b), ou par d'autres substances associées à la biosynthèse de la phéromone proprement dite (Frenoy et al. 1992). D'autres utilisent les odeurs émanant des écailles laissées par la femelle sur le substrat après la ponte
(Zaborski et al. 1987), ou des oeufs pondus (Renou et al. 1992). Pour certains entomophages, les fèces de larves de Lépidoptères sont un autre stimulus important (Auger et al. 1989; Eller et al. 1988). La recherche de I'hôte peut aussi faire intervenir trois niveaux de la chaîne trophique comme c'est le cas chez les femelles du parasitoïde généraliste, Cotesia marginiventris (Cresson) [Hymenoptera: Braconidae]; celles-ci répondent peu ou pas aux plantes intactes ou artificiellement attaquées, ni aux odeurs des larves ou de leurs fèces, mais sont particulièrement attirées par les plantes attaquées par l'hôte. Plus précisément, les terpènes agissant comme kairomone, sont produits lorsque les sécrétions orales des larves viennent en contact avec les cellules végétales endommagées (Turlings et al. 1990a, 1991a, 1991b). Des tests de préférence ont montré aussi que les femelles de ce parasitoïde étaient capables de différencier les arômes produits par différentes espèces de larves sur une plante donnée ou celles produites par la même larve sur différentes plantes (Turlings et al. 1990b).

Comprendre le rôle des infochimiques intervenant dans les relations entre phytophages et entomophages peut être utile aussi pour créer des conditions optimales d'élevage de certains agents de lutte biologique. Par exemple, le vol et la ponte du Rhizophagus grandis (Gyll.) [Coleoptera: Rhyzophagidae], un prédateur européen du dendroctone de l'écorce, Dendroctonus micans (Kug.) [Coleoptera: Scolytidae], augmentent en présence des fèces de sa proie (Grégoire et al. 1991, 1992). C'est grâce à I'utilisation de ces volatiles que la production massive du $R$. grandis en laboratoire est devenue plus facile et moins coûteuse (Grégoire et al. 1992). Cette méthode d'élevage facilitera non seulement la lutte biologique contre le $D$. micans en Europe, mais sera aussi utile advenant l'introduction du $R$. grandis en Amérique du Nord pour combattre le Dendroctonus valens (Lec.) [Coleoptera: Scolytidae], un dendroctone très voisin du $D$. micans, mais dépourvu d'ennemis naturels indigènes sur notre continent (Grégoire et al. 1992).

En nature, plusieurs espèces de parasitoïdes peuvent, dès leur émergence, être 
exposés à différents infochimiques comme ceux émanant des derniers aliments ingérés par l'hôte et présents, dans le cocon, sous forme d'excréments. La reconnaissance de ces odeurs (par association) peut aider les parasitoïdes à trouver plus efficacement leurs hôtes potentiels (Lewis et Tumlinson 1988; Lewis et al. 1991); d'ailleurs certains parasitoïdes sont peu attirés par les odeurs des plantes défoliées s'ils ne sont pas préalablement exposés à ces odeurs (Hérard et al. 1988a, 1988b; Turlings et al. 1990b). Comme les hôtes utilisés pour la production massive de larves parasitoïdes sont généralement élevés sur diète artificielle plutôt que sur du matériel végétal, et ce, pour des raisons budgétaires ou d'espace, les parasitoïdes ne sont en conséquence jamais exposés aux odeurs des plantes avant d'être relâchés sur le terrain. Comme ceci peut affecter l'efficacité de recherche des entomophages, du moins initialement, il s'agirait alors de les exposer pendant une brève période aux arômes des plantes-hôtes, afin d'augmenter leur performance dans les programmes de lutte biologique.

Le miellat qu'excrètent les pucerons sous forme d'un mélange complexe de sucres, de sels et d'acides aminés a pour effet de stimuler, chez certains entomophages, la recherche de I'hôte ainsi que la ponte (Bouchard et Cloutier 1984; Budenberg 1990; Budenberg et Powell 1992; Budenberg et al. 1992). Cependant, une solution simple de sucre ne favorise pas, chez le parasitoïde Aphidius nigripes (Ashmead) [Hymenoptera: Aphidiidae], une recherche intensive de son hôte, le Macrosiphum euphorbiae (Thomas) [Homoptera: Aphididae] (Bouchard et Cloutier 1984): par contre, un effet plus significatif a été obtenu chez l'Aphidius rhopalosiphi (De Stefani-Perez) [Hymenoptera: Aphidiidae] utilisant un miellat artificiel de composition complexe (Budenberg 1990). Donc, en principe, les infochimiques attractifs aux prédateurs et parasitoïdes pourraient être utilisés pour augmenter leur impact sur les insectes nuisibles, sans affecter le milieu. Cependant, comme les parasitoïdes s'habituent très rapidement aux stimuli olfactifs et, qu'en absence d'hôtes, ils ont tendance à quitter I'habitat, I'utilisation de ces substances, dans une perspective de lutte intégrée, pourrait être en soi limitée (Bouchard et Cloutier 1984; Budenberg 1990).

\section{LA SIMPLICITÉ N'EST PAS TOUJOURS LA RËGLE}

Les plantes qui font "appel à des gardes du corps" dès qu'elles sont attaquées par un insecte ne concernent pas que les parasitoïdes, puisque ce phénomène a été observé aussi entre des acariens phytophages et prédateurs (Dicke et Sabelis 1988, 1992; Dicke et al. 1990). II existe aussi des exemples démontrant que les plantes défoliées peuvent être à la fois attirantes à l'entomophage et inacceptables au phytophage comme source de nourriture (Turlings et Tumlinson 1991). Également, des plantes non attaquées mais contaminées par les odeurs de plantes défoliées peuvent devenir moins convenables pour la ponte à l'acarien phytophage et à la fois plus attirantes pour l'acarien prédateur, que des plantes intactes et non contaminées (Bruin et al. 1992). Bien que ces études démontrent que la phytophagie induit la défense chimique chez les plantes et favorise l'attraction des ennemis naturels, il y a des cas où la production de ces substances défensives peut être désavantageuse. Par exemple, en réponse à une forte défoliation causée par les larves de la spongieuse [Lymantria dispar (L.)] [Lepidoptera: Lymantriidae], la teneur en phénols du chêne rouge (Quercus rubra L.) subit une hausse (Schultz et Baldwin 1982) dont l'effet se traduit par une diminution du poids des pupes, et par conséquent, la fécondité des femelles (Rossiter et al. 1988). Cependant, I'augmentation des tannins solubles, résultant de la défoliation, inhibe l'efficacité du virus de la polyhédrose nucléaire (Keating et al. 1990; Schultz et al. 1990), un important facteur de mortalité des larves de la spongieuse. Ceci montre bien comment la défense chimique de l'arbre peut offrir des conditions favorables à l'expansion de la population de l'insecte nuisible. Ainsi, la connaissance de ces interactions chimiques permet non seulement de mieux expliquer les fluctuations de la densité des populations, comme c'est le cas chez la spongieuse (Foster et al. 1992), mais aussi de mieux planifier 
I'utilisation du virus dans les programmes de lutte biologique.

Les scénarios dans lesquels "ce que l'un perd, I'autre le gagne" sont aussi rencontrés dans les agro-écosystèmes. Chez la tomate sauvage (Lycopersicon hirsutum f. glabratum C. H. Mull) par exemple, la résistance chimique, associée à la présence de trichomes, devient plus faible suite à la fertilisation des plantes; le traitement a pour effet de réduire la densité des trichomes ainsi que les kétones méthylés qu'ils contiennent (Barbour et al. 1991). Cependant, cette résistance réduite pourrait éventuellement être contrebalancée par une augmentation de l'efficacité des entomophages, vu que ces derniers sont affectés négativement par la présence des trichomes et de leur contenu chimique (Kauffman et Kennedy 1989a, 1989b, 1989c).

\section{CONCLUSION}

Bien que brève et quelque peu sélective, nous espérons que cette revue de littérature aura su démontrer que la recherche en écologie chimique ne génère pas que des informations fondamentales sur la nature complexe et fascinante des systèmes biologiques mais qu'elle offre aussi des solutions plus intelligentes et naturelles de lutter contre les insectes nuisibles. Cependant, afin de mieux promouvoir ce potentiel, il serait important que les chercheurs cessent de faire des comparaisons stériles entre les avantages relatifs de la recherche fondamentale et appliquée et collaborent ensemble à la réalisation d'un objectif commun.

\section{REMERCIEMENTS}

Nous remercions Benoît Arsenault, André Bouchard, Jacques Brodeur, Michel Cusson et Martin Hardy pour les conseils judicieux apportés lors de la révision de cet article.

\section{RÉFÉRENCES}

Aluja, M. et E. F. Boller. 1992. Host marking pheromone of Rhagoletis cerasi: field deployment synthetic pheromone as a novel cherry fruit fly management strategy. Entomol. Exp. Appl. 65: 141-147.

Auger, J., C. Lecomte, J. Paris et E. Thibout. 1989. Identification of leek-moth and diamondback-moth frass volatiles that stimulate parasitoid, Diadromus pulchellus. J. Chem. Ecol. 15: 1391-1398.

Averill, A. L. et R. J. Prokopy. 1987. Residual activity of oviposition-deterring pheromone in Rhagoletis pomonella (Diptera:Tephritidae) and female response to infested fruit. J. Chem. Ecol. 13: 167-177.

Barbosa, P. et D. K. Letourneau (réds.). 1988. Novel aspects of insect-plant interactions. Wiley Interscience, New York. 362 pp.

Barbour, J. D., R. R. Farrar Jr. et G. G. Kennedy. 1991. Interaction of fertilizer regime with host-plant resistance in tomato. Entomol. Exp. Appl. 60: 301-304.

Birch, M. C., G. M. Poppy et T. C. Baker. 1990. Scents and eversible scent structures of male moths. Annu. Rev. Entomol. 35: 2558.

Bouchard, Y. et C. Cloutier. 1984. Honeydew as a source of host-searching kairomones for the aphid parasitoid Aphidius nigripes (Hymenoptera: Aphidiidae). Can. J. Zool. 62: 1513-1520.

Bruin, J., M. Dicke et M. W. Sabelis. 1992. Plants are better protected against spidermites after exposure to volatiles from infested conspecifics. Experientia (Basel) 48: 525-529.

Budenberg, W. J. 1990. Honeydew as a contact kairomone for aphid parasitoids. Entomol. Exp. Appl. 55: 139-148.

Budenberg, W. J. et W. Powell. 1992. The role of honeydew as an ovipositional stimulant for two species of syrphids. Entomol. Exp. Appl. 64: 57-61.

Budenberg, W. J., W. Powell et S. J. Clark. 1992. The influence of aphids and honeydew on the leaving rate of searching aphids parasitoids from wheat plants. Entomol. Exp. Appl. 63: 259-264.

Cardé, R. T. 1990. Principles of mating disruption. Pages $9-23$ in R. L. Ridway, R. M. Silverstein et M. N. Inscoe (réds.), Behavior-modifying chemicals for insect management: applications of pheromones and other attractants. Marcel Dekker, New York.

Chapman, R. F. et E. A. Bernays. 1989. Insect behavior at the leaf surface and learning as aspects of host plant selection. Experientia (Basel) 45: 215-222. 
Delisle, J. 1992. Monitoring the seasonal male flight activity of Choristoneura rosaceana (Lepidoptera: Tortricidae) in eastern Canada using virgin females and several different pheromone blends. Environ. Entomol. 21: 1007-1012.

Delisle, J., J. N. McNeil, E. W. Underhill et D. Barton. 1989. Helianthus annuus pollen, an oviposition stimulant for the sunflower moth, Homoeosoma electellum. Entomol. Exp. Appl. 50: 53-60.

Dicke, M. et M. W. Sabelis. 1988. Infochemical terminology: based on cost-benefit analysis rather than origin of compounds? Funct. Ecol. 2: 131-139.

Dicke, M. et M. W. Sabelis. 1992. Costs and benefits of chemical information conveyance: proximate and ultimate factors. Pages 122-155 in B. D. Roitberg et M. B. Isman (réds.), Insect chemical ecology: an evolutionary approach. Chapman and Hall, NewYork.

Dicke, M., M. W. Sabelis, J. Takabayashi, J. Bruin et M. A. Posthumus. 1990. Plant strategies of manipulating predator-prey interactions through allelochemicals: prospects for application in pest control. J. Chem. Ecol. 16: 3091-3118.

Dussourd, D. E., K. Ubik, C. Harvis, J. Resch, J. Meinwald et T. Eisner. 1988. Biparental defensive endowment of eggs with acquired plant alkaloid in the moth Utetheisa ornatrix. Proc. Nat. Acad. Sci. USA 85: 59925996.

Eller, F. J., J. H. Tumlinson et J. W. Lewis. 1988. Beneficial arthropod behavior mediated by airborne semiochemicals: sources of volatiles mediating the host-location flight behavior of Microplitis croceipes (Cresson) (Hymenoptera: Braconidae), a parasitoid of Heliothis zea (Boddie) (Lepidoptera: Noctuidae). Environ. Entomol. 17: 745-753.

Feeny, P. 1992. The evolution of chemical ecology: contributions from the study of herbivorous insects. Pages 1-44 in G. A. Rosenthal et M. R. Berenbaum (réds.), Herbivores: their interactions with secondary plant metabolites. Vol II. Evolutionary and ecological processes. Academic Press, New York.

Fitzpatrick, S. M. et J. N. McNeil. 1988. Male scent in lepidopteran communication: The role of male pheromone in mating behaviour of Pseudaletia unipuncta (Haw.) (Lepidoptera:Noctuidae). Pages 131-151 in T. S. Sahota et C. S. Holling (réds.), Paths from a viewpoint: the Wellington festschrift on insect ecology. Mem. Entomol. Soc. Can. 146.
Foster, M. A., J. C. Schultz et M. D. Hunter. 1992. Modelling gypsy moth-virus-leaf chemistry interactions: implications of plant quality for pest and pathogen dynamics. J. Anim. Ecol. 61: 509-520.

Frenoy, C., C. Durier et N. Hawlitzky. 1992. Effect of kairomones from egg and female adult stages of Ostrina nubilalis (Hübner) (Lepidoptera: Pyralidae) on Trichogramma brassicae Bezdenko (Hymenoptera: Trichogrammatidae) female kinesis. J. Chem. Ecol. 18: 761-773.

Gilbert, L. E. 1972. Pollen feeding and reproductive biology of Heliconius butterflies. Proc. Nat. Acad. Sci. USA 69: 1403-1407.

Grégoire, J. C., M. Baisier, A. Drumont, D. L. Dahlsten, H. Meyer et W. Francke. 1991. Volatile compounds in the larval frass of Dendroctonus valens and Dendroctonus micans (Coleoptera: Scolytidae) in relation to oviposition by the predator, Rhizophagus grandis (Coleoptera: Rhizophagidae). J. Chem. Ecol. 17: 2003-2019.

Grégoire, J. C., D. Couillien, R. Krebber, W. A. Konig, H. Meyer et W. Francke. 1992. Orientation of Rhizophagus grandis (Coleoptera: Rhizophagidae) to oxygenated monoterpenes in a species-specific predator-prey relationship. Chemoecology. 3: 1418.

Hardee, D. D., W. H. Cross, E. B. Mitchell, P. M. Huddleston, H. C. Mitchell, M. E. Merkl et T. B. Davich. 1969. Biological factors influencing responses of the female boll weevil to the male sex pheromone in field and large-cage tests. J. Econ. Entomol. 62: $161-165$.

Haynes, K. F. et T. C. Baker. 1989. An analysis of amenotactic flight in female moths stimulated by host odor and comparison with the males' response to sex pheromone. Physiol. Entomol. 14: 279-289.

Haynes, K. F., W.-G. Li et T. C. Baker. 1986a. Control of pink bollworm moth (Lepidoptera: Gelechiidae) with insecticides and pheromones (attracticide): lethal and sublethal effects. J. Econ. Entomol. 79: 1466-1471.

Haynes, K. F., T. A. Miller, R. T. Staten, W.-G. Li et T. C. Baker. 1986b. Monitoring insecticide resistance with insect pheromones. Experientia (Basel) 42: 1293-1295.

Haynes, K. F., T. A. Miller, R. T. Staten, W.-G. Li et T. C. Baker. 1987. Pheromone trap for monitoring insecticide resistance in the pink bollworm moth (Lepidoptera: Gelechiidae): new tool for resistance management. Environ. Entomol. 16: 84-89.

Haynes, K. F., J. Z. Zhao et A. Latif. 1991. Identification of floral compounds from Abelia grandiflora that stimulate upwind flight in cabbage looper moths. J. Chem. Ecol. 17: 637-646. 
Heinrichs, E. A. (réd.). 1988. Plant stressinsect interactions. Wiley Interscience, New York. 492 pp.

Hérard, F., M. A. Keller, W. J. Lewis et J. H. Tumlinson. 1988a. Beneficial arthropod behavior mediated by airborne semiochemicals. III. Influence of age and experience on flight chamber responses of Microplitis demolitor Wilkinson. J. Chem. Ecol. 14: 1583-1596.

Hérard, F., M. A. Keller, W. J. Lewis et J. H. Tumlinson. 1988b. Beneficial arthropod behavior mediated by airborne semiochemicals. IV. Influence of host diet on hostoriented flight chamber responses of $\mathrm{Mi}$ croplitis demolitor Wilkinson. J. Chem. Ecol. 14: 1597-1606.

Hunter, M. D., T. Ohgushi et P. W. Price (réds.). 1992. Effects of resource distribution on animal-plant interactions. Academic Press, New York. 505 pp.

Hurter, J., E. F. Boller, E. Stadler, B. Blattmann, H. -R. Buser, N. U. Bosshard, L. Damm, M. W. Kozlowski, R. Schoni, F. Raschdorf, R. Dahinden, E. Schlumpf, H. Fritz, W. J. Richter et J. Schreiber. 1987. Oviposition-deterring pheromone in Rhagoletis cerasi L.: purification and determination of the chemical constitution. Experientia (Basel) 43: 157-164.

Jutsum, A. R. et R. F. S. Gordon, (réds.). 1989. Insect pheromones in plant protection. John Wiley, New York. 369 pp.

Kauffman, W. C. et G. G. Kennedy. 1989a. Relationship between trichome density in tomato and parasitism of Heliothis spp. (Lepidoptera: Noctuidae) eggs by Trichogramma spp. (Hymenoptera: Trichogrammatidae). Environ. Entomol. 18: 698-704.

Kauffman, W. C. et G. G. Kennedy. 1989b. Inhibition of Campoletis sonorensis parasitism of Heliothis zea and of parasitoid development by 2-tridecanone-mediated insect resistance of wild tomato. J. Chem. Ecol. 15: 1919-1930.

Kauffman, W. C. et G. G. Kennedy. 1989c. Toxicity of allelochemicals from wild insect-resistant tomato Lycopersicon hirsutum f. glabratum to Campoletis sonorensis, a parasitoid of Heliothis zea. J. Chem. Ecol. 15: 2051-2060.

Keating, S. T., M. D. Hunter et J. C. Schultz. 1990. Leaf phenolic inhibition of gypsy moth nuclear polyhedrosis virus: role of polyhedral inclusion body aggregation. J. Chem. Ecol. 16: 1445-1457.

Klijnstra J. W. et L. M. Schoonhoven. 1987. Effectiveness and persistence of the oviposition deterring pheromone of Pieris brassicae in the field. Entomol. Exp. Appl. 40: 71-79.
Kuba, H. et Y. Sokei. 1988. The production of pheromone clouds by spraying in the melon fly, Dacus cucurbitae Coquillett (Diptera: Tephritidae). J. Ethol. 6: 105-110.

Landolt, P. J. 1989. Attraction of the cabbage looper to host plants and host plant odor in the laboratory. Entomol. Exp. Appl. 53: 117-124.

Landolt, P. J. et R. R. Heath. 1990. Sexual role reversal in mate-finding strategies of the cabbage looper moth. Science (Wash. DC) 249: 1026-1028.

Landolt, P. J., B. Lenczewski et R. R. Heath. 1991. Lure and toxicant system for the cabbage looper (Lepidoptera: Noctuidae). J. Econ. Entomol. 84: 1344-1347.

Lanier, G. N. 1990. Principles of attractionannihilation: mass trapping and other means. Pages 25-45 in R. L. Ridway, R. M. Silverstein et M. N. Inscoe (réds.), Behavior-modifying chemicals for insect management: applications of pheromones and other attractants. Marcel Dekker, New York.

Lewis, W. J. et J. H. Tumlinson. 1988. Host detection by chemically mediated associative learning in a parasitic wasp. Nature (Lond.) 331: 257-259.

Lewis, W. J., J. H. Tumlinson et S. Krasnoff. 1991. Chemically mediated associative learning: an important function in the foraging behavior of Microplitis croceipes (Cresson). J. Chem. Ecol. 17: 1309-1325.

McNeil, J. N. 1991. Behavioral ecology of pheromone-mediated communication in moths and its importance in the use of pheromone traps. Annu. Rev. Entomol. 36: 407-430.

McNeil, J. N. 1992. Evolutionary perspectives and insect pest control: an attractive blend for the deployment of semiochemicals in management programs. Pages 334-351 in B. D. Roitberg et M. B. Isman (réds.), Insect chemical ecology: an evolutionary approach. Chapman and Hall, New York.

Metcalf, R. L. 1980. Changing role of insecticides in crop protection. Annu. Rev. Entomol. 25: 219-256.

Metcalf, R. L et E. R. Metcalf. 1992. Plant kairomones in insect ecology and control. Chapman and Hall, New York. 168 pp.

Muirhead-Thomson, R. C. 1991. Trap responses of flying insects. The influence of trap design on capture efficiency. Academic Press, New York. 287 pp.

Noldus, L. P. J. J., J. C. van Lenteren et W. J. Lewis. 1991a. How Trichogramma parasitoids use moth sex pheromones as kairomones: orientation behavior in a wind tunnel. Physiol. Entomol. 16: 313-327. 
Noldus, L. P. J. J., R. P. J. Potting et H. E. Barendregt. 1991b. Moth sex pheromone adsorption to leaf surface: bridge in time for chemical spies. Physiol. Entomol. 16: 329-344.

Phelan, P. L. et T. C. Baker. 1986. Male-sizerelated courtship success and intersexual selection in the tobacco moth, Ephestia elutella. Experientia (Basel) 43: 1291-1293.

Pivnick, K. A., J. Lavoie-Dornik et J. N. McNeil. 1992. The role of the androconia in the mating behaviour of the European skipper, Thymelicus lineola, and evidence for a male sex pheromone. Physiol. Entomol. 17: 260-268.

Price, P. W., T. M. Lewinsohn, G. W. Fernades et W. W. Benson (réds.). 1992. Plant-animal interactions: Evolutionary ecology in tropical and temperate regions. Wiley Interscience, New York. 639 pp.

Quiring, D. T. et J. N. McNeil. 1984. Intraspecific competition between different aged larvae of Agromyza frontella (Rondani) (Diptera: Agromyzidae): advantages of an oviposition-deterring pheromone. Can. J. Zool. 62: 2192-2196.

Quiring, D. T. et J. N. McNeil. 1987. Foraging behavior of a dipteran leaf miner on exploited and unexploited hosts. Oecologia (Heidelb.) 73: 7-15.

Rabb, R. L. et F. E. Guthrie (réds.). 1970. Concepts of pest management. North Carolina State University, Raleigh, NC. 242 pp.

Ramaswamy, S. B. 1988. Host finding by moths: Sensory modalities and behaviours. J. Insect Physiol. 34: 235-249.

Renou, M., P. Nagnan, A. Berthier et C. Durier. 1992. Identification of compounds from the eggs of Ostrinia nubilalis and Mamestra brassicae having kairomone activity on Trichogramma brassicae. Entomol. Exp. Appl. 63: 291-303.

Renwick, J. A. A. 1989. Chemical ecology of oviposition in phytophagous insects. Experientia (Basel) 45: 223-228.

Renwick, J. A. A., C. D. Radke, K. SachdevGupta et E. Stadler. 1992. Leaf surface chemicals stimulating oviposition by Pieris rapae (Lepidoptera: Pieridae) on cabbage. Chemoecology 3: 33-38.

Ridway, R. L., R. M. Silverstein et M. N. Inscoe (réds.). 1990. Behavior-modifying chemicals for insect management: applications of pheromones and other attractants. Marcel Dekker, New York. 761 pp.

Roitberg, B. D. et M. B. Isman (réds.). 1992. Insect chemical ecology: an evolutionary approach. Chapman and Hall, New York. $359 \mathrm{pp}$
Roitberg, B. D., J. C. van Lenteren, J. J. M. van Alphen, F. Galis et R. J. Prokopy. 1982. Foraging behaviour of Rhagoletis pomonella a parasite of hawthorn (Crataegus viridis), in nature. J. Anim. Ecol. 51: 307325.

Roitberg, B. D., R. S. Cairl et R. J. Prokopy. 1984. Oviposition deterring pheromone influences dispersal distance in tephritid fruit flies. Entomol. Exp. Appl. 35: 217-220.

Rossiter, M. C., J. C. Schultz et I. T. Baldwin. 1988. Relationships among defoliation, red oak phenolics, and gypsy moth growth and reproduction. Ecology 69: 267-277.

Royer, L. et J. N. McNeil. 1992. Evidence of a male sex pheromone in the European corn borer, Ostrinia nubilalis (Hübner) (Lepidoptera: Pyralidae). Can. Entomol. 124: 113116.

Royer, L. et J. N. McNeil. 1993. Male investment in the European corn borer, Ostrinia nubilalis (Hübner) (Lepidoptera: Pyralidae): impact on female longevity and reproductive performance. Funct. Ecol. 7: 209-215.

Schoonhoven, L. M. 1990. Host-marking pheromones in Lepidoptera, with special reference to two Pieris spp. J. Chem. Ecol. 16: 3043-3052.

Schoonhoven, L. M., E. A. M. Beerling, J. W. Klijnstra et Y. van Vugt. 1990. Two related butterfly species avoid oviposition near each other's eggs. Experientia (Basel) 46: 526-528.

Schultz, J. C. et I. T. Baldwin. 1982. Oak leaf quality declines in response to defoliation by gypsy moth larvae. Science (Wash. DC) 217: 149-151.

Schultz, J. C., M. A. Foster et M. E. Montgomery. 1990. Host plant-mediated impact of a baculovirus on gypsy moth populations. Pages 303-313 in A. D. Watt, S. R. Leather, M. D. Hunter, et N. A. C. Kidd (réds.), Population dynamics of forest insects. Intercept, Andover.

Smith, C. M. (réd.). 1989. Plant resistance to insects: a fundamental approach. Wiley Interscience, New York. 286 pp.

Tingle, F. C. et E. R. Mitchell. 1991. Effect of oviposition deterrents from elderberry on behavioral responses by Heliothis virescens to host-plant volatiles in flight tunnel. J. Chem. Ecol. 17: 1621-1631

Tingle, F. C., E. R. Mitchell et R. R. Heath. 1990. Preferences of mated Heliothis virescens and $H$. subflexa females for host and nonhost volatiles in a flight tunnel. J. Chem. Ecol. 16: 2889-2898.

Turlings, T. C. J. et J. H. Tumlinson. 199\%. Do parasitoids use herbivore-induced plant chemical defenses to locate host? Fla. Entomol. 74: 42-50. 
Turlings, T. C. J., J. W. A. Scheepmaker, L. E. M. Vet, J. H. Tumlinson et W. J. Lewis. 1990a. How contact foraging experiences affect preferences for host-related odors in the larval parasitoid Cotesia marginiventris (Cresson) (Hymenoptera: Braconidae). J. Chem. Ecol. 16: 1577-1589.

Turlings, T. C. J., J. H. Tumlinson et W. J. Lewis. 1990b. Exploitation of herbivoreinduced plant odors by host-seeking parasitic wasps. Science (Wash. DC) 250: 12511253.

Turlings, T. C. J., J. H. Tumlinson, F. J. Eller et W. J. Lewis. 1991a. Larval-damaged plants: source of volatile synomones that guide the parasitoid Cotesia marginiventris to the micro-habitat of its hosts. Entomol. Exp. Appl. 58: 75-82.

Turlings, T. C. J., J. H. Tumlinson, R. R. Heath, A. T. Proveaux et R. E. Doolittle. 1991b. Isolation and identification of allelochemicals that attract the larval parasitoid, Cotesia marginiventris (Cresson), to the microhabitat of one of its hosts. J. Chem. Ecol. 17: 2235-2251.

van Loon, J. J., A., A. Blaakmeer, F. C. Griepink, T. A. van Beek, L. M. Schoonhoven et A. de Groot. 1992. Leaf surface compound from Brassica oleracae (Cruciferae) induces oviposition by Pieris brassicae (Lepidoptera: Pieridae). Chemoecology. 3: 39-44.
Visser, J. H. 1986. Host odor perception in phytophagous insects. Annu. Rev. Entomol. 31: 121-144.

Wall, C. 1990. Principles of monitoring. Pages. 9-23 in R. L. Ridway, R. M. Silverstein et M. N. Inscoe (réds.), Behavior-modifying chemicals for insect management: applications of pheromones and other attractants. Marcel Dekker, New York.

Wiesenborn, W. D. et T. C. Baker. 1990. Upwind flight to flowers by Pectinophora gossypiella (Lepidoptera: Gelechiidae). Environ. Entomol. 19: 490-493.

Willis, M. A. et M. C. Birch. 1982. Male lek formation and female calling in a population of the arctiid moth Estigmene acrea. Science (Wash. DC) 218: 168-170.

Zaborski, E., P. E. A. Teal et J. E. Laing. 1987. Kairomone-mediated host finding by spruce budworm egg parasite, Trichogramma minutum J. Chem. Ecol. 13: 113-122 